\title{
Level 7: Erste Schritte zur ultimativen Gaming-Bibliothek
}

Sie haben nun das nächste Level des Abenteuers „Gaming in Bibliotheken“ erreicht. Sie haben viel gelernt. Sie wissen nun, was mit dem Begriff Gaming gemeint ist. Sie wissen, dass Gaming kein reines Kinder- und Jugendthema ist, sondern dass diese Kultur in allen Altersgruppen große Fangruppen hat. Sie wissen, dass Gaming mehr ist als nur ein sinnloses Freizeitvergnügen. Sie wissen, dass Gaming Lernen bedeutet. Sie haben verschiedene Genres kennengelernt und wissen auch, wie die aktuellen Vertriebsmodelle aussehen. Sie kennen nun die Grenzen der klassischen Bestandsarbeit und Sie haben einige Ansätze für weitergehende Aktivitäten einer Bibliothek im Bereich Gaming kennengelernt. Nun müssen Sie sich überlegen, wie Sie sich dem Thema strategisch am besten nähern. Aus diesem Grund geht es im siebten Level um die Entwicklung einer Gaming-Strategie für eine Bibliothek. Ich kann Ihnen keine Gebrauchsanweisung geben. Jede Bibliothek wird ihre eigene Strategie entwickeln und realisieren müssen. Es gibt keine fertigen Konzepte. Dafür sind Bibliotheken zu unterschiedlich. Die Unterschiede ergeben sich weniger aus den Ressourcen und Strukturen als vielmehr aus den Menschen, die in der Bibliothek arbeiten, den Menschen, die Kunden der Bibliothek sind und den Menschen, die zum Umfeld der Bibliothek gehören. Insofern sollen die nun folgenden Ansätze, Überlegungen und Tipps als Inspiration bzw. als Hilfestellung dienen.

\section{Grundsätzliche Überlegungen}

Egal für welche Varianten und Projekte Sie sich entscheiden, eines wird nicht möglich sein: Sie können nicht „ein bisschen was mit Gaming machen“. Dieser Ansatz kann nicht funktionieren, denn dafür ist das Thema zu komplex und die Zielgruppe zu heterogen. Gaming muss die gleiche Relevanz wie das Lesen eines Buches oder irgendeine andere Aktivität in Ihrer Bibliothek haben. Das bedeutet nicht, dass Sie auch die gleiche Menge an Ressourcen für dieses Thema aufbringen müssen, aber es darf niemals ein Thema sein, welches nur dann angegangen wird, wenn die anderen Aufgaben der Bibliothek abgeschlossen bzw. erledigt sind. Gaming ist auch kein Mittel, um Menschen in die Bibliothek zu locken, damit diese dann zum Lesen von Büchern animiert werden können. Gaming ist Gaming. Gaming ist Lernen, Lesen, Spielen, Spaß haben, Wissen teilen, Wissen erschließen, Kommunikation, Interaktion, Feedback etc. All das bedeutet Gaming und all das sollte Gaming auch in Ihrer Bibliothek sein.

Gaming wird Ihre Bibliothek verändern. Es wird dafür sorgen, dass Sie neue Zielgruppen erreichen. Aber wollen Sie diese Zielgruppen überhaupt erreichen? Wie gesagt, es geht nicht darum, Gaming als Werbeplattform für Ihre anderen Angebote zu missbrauchen. Erfolg im Bereich Gaming wird nicht durch Masse, sondern durch Klasse erreicht. Es geht also nicht darum, alle von mir beschriebenen Angebote umzusetzen. Vielmehr sollten Sie die Angebote auswählen oder eigene Angebote entwickeln, die zu Ihnen als Bibliothek passen und die Sie umsetzen können.

Ihnen muss aber auch klar sein, dass die Arbeit mit dem Thema Gaming ein Versprechen ist. Sie versprechen damit, dass Sie sich auf die Gaming-Kultur einlassen wollen und dass Sie dieses Thema nicht nur angehen, um damit neue Leser zu generieren.

Mir ist wichtig, dass Sie sich diese eben genannten Punkte noch einmal durch den Kopf gehen lassen. Reden Sie am besten auch mit dem ganzen Team darüber, denn 
Sie treffen eine Entscheidung, die einiges an Ihrer Arbeit ändern wird. Ich möchte kurz auf ein paar Punkte eingehen, die diese Änderung sehr gut beschreiben:

1. Mit dem Thema Gaming bearbeiten Sie einen Bereich, dessen Nutzung sich von allen anderen Medien unterscheidet. Games werden nicht konsumiert, Games werden aktiv gespielt.

2. Die Gaming-Kultur ist eine ganz eigene Kultur und sie ist älter als die meisten Menschen glauben.

3. Games sind Innovationsträger. Sie stehen für moderne Hard- und Software und die damit verbundene Nutzung.

4. Games sind ein elementarer Bestandteil der digitalen Welt. Damit haben sie eine tiefgreifende Auswirkung auf die Bibliotheksarbeit. Ein Beispiel: Wie bereits beschrieben, lassen sich Games nur bedingt in eine klassische Bestandsstrategie aufnehmen. Die zunehmende Digitalisierung macht dies zunehmend unmöglich. Dieser Prozess ist aber nicht auf die Games beschränkt. Vielmehr werden wir erleben, dass auch Bücher, Filme und Musik mit der fortschreitenden Digitalisierung einen tiefgreifenden Wandel erleben werden. Anders ausgedrückt: Was Sie im Bereich Gaming lernen, indem Sie sich z.B. weniger auf den Bestand und mehr auf Services fokussieren, werden Sie sehr bald auch in anderen Bereichen anwenden können.

5. Games sind wild, laut und nicht unumstritten. Und Games erfordern es, dass Sie als Bibliotheksmitarbeiter spielen.

Wenn Sie gemeinsam diese Punkte besprochen haben, kann es losgehen mit der Entwicklung und Realisierung einer Gaming-Strategie.

\section{Zielsetzungen}

Bevor Sie mit eigenen Aktivitäten starten, sollten Sie sich zuerst überlegen, warum Sie sich mit dem Thema beschäftigen wollen bzw. wer Ihre Zielgruppe sein soll. In meinen Workshops erlebe ich oft, dass man sich hinsichtlich der Frage des „Warum?“ und „Für Wen?“ zu wenig Gedanken gemacht hat.

Sie haben in diesem Buch viele Argumente gehört, warum meiner Meinung nach Bibliotheken etwas mit dem Thema Gaming anfangen sollten. Aber vielleicht passen all diese Punkte nicht zu Ihnen? Oder haben Sie vielleicht weitere Gründe und Zielsetzungen gefunden? Sammeln Sie die Zielsetzungen in einer Matrix. Dabei können Sie auch schon mögliche Projekte, Zielgruppen und benötigte Ressourcen eintrage. Diese Matrix könnte so aussehen:

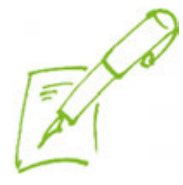

\begin{tabular}{llll}
\hline Zielsetzung & Mögliche Projekte & Zielgruppe & Benötigte Ressourcen \\
\hline $\begin{array}{l}\text { Leseförderung } \\
\text { durch Games }\end{array}$ & $\begin{array}{l}\text { Storytelling, } \\
\text { Let's Play }\end{array}$ & $\begin{array}{l}\text { Jugendliche zwischen } \\
12-16 \text { Jahren }\end{array}$ & $\begin{array}{l}\text { Spiele, Konsolen, } \\
\text { Software, Zeit, Raum }\end{array}$ \\
\hline etc. & etc. & etc. & etc. \\
\hline etc. & etc. & etc. & etc. \\
\hline etc. & etc. & etc. & etc. \\
\hline etc. & etc. & etc. & etc. \\
\hline
\end{tabular}


Diese Sammlung ist sehr wichtig, denn Sie wird Ihnen nicht nur als Vorlage für Ihre zukünftige Arbeit dienen. Sie ist auch eine gute Hilfe bei Diskussionen. Sie erschaffen sich eine Argumentationsvorlage, die Sie immer wieder erweitern und verändern können.

\section{Zielgruppendefinition}

Ich habe schon an verschiedenen Stellen in diesem Buch auf die Frage der Zielgruppen hingewiesen. Es geht bei der Frage nach den Zielgruppen weniger um eine Fokussierung auf eine einzelne Gruppe, sondern vielmehr um das Herausarbeiten der Bedürfnisse und Hintergründe der jeweiligen Zielgruppe. So benötigen erwachsene Gamer andere Zeiten und Inhalte als Kinder oder Jugendliche. Ausschließen sollten Sie in diesem Stadium keine der Gamer-Gruppen.

Vergessen Sie auf keinen Fall die Zielgruppe der weiblichen Gamer. Gaming ist nicht nur etwas für Jungs. Es ist ebenso ein Thema für Mädchen. Es kann sinnvoll sein, spezielle Events für Mädchen und weibliche Jugendliche zu entwickeln, bei denen männliche Gamer keinen Zutritt haben.

Wenn Sie die Zielgruppen definiert haben, können Sie damit beginnen zu überlegen, was diese Gruppe interessieren könnte. Und dann kommt der wichtigste Punkt: Sie müssen überlegen, was Ihre Bibliothek zu einem einzigartigen Gaming-Ort macht. Denken Sie immer daran: Gamer können ihre Spiele auch zu Hause spielen. Sie brauchen dafür keine Bibliothek. Insofern müssen Sie ein Konzept entwickeln, welches die Bibliothek zu einem besonderen Spielort macht.

Wenn Sie diesen Punkt abgeschlossen haben, müssen Sie überlegen, wie Ihre aktuelle Situation aussieht, denn das ist die Basis für alle zukünftigen Aktivitäten.

\section{Analyse des Ist-Zustands}

Wie bei allen Aktivitäten ist es auch bei diesem Thema wichtig, zuerst eine Analyse des Ist-Zustands durchzuführen. Dabei geht es nicht nur um eine Analyse der vorhandenen technischen Ressourcen. Vielmehr sollten Sie das ganze System Bibliothek hinsichtlich der Frage analysieren, ob Sie überhaupt in der Lage sind, mit dem Thema Gaming zu arbeiten. Im Rahmen dieser Analyse werden Sie feststellen, dass Sie es mit einigen Fragestellungen zu tun haben, die auch in anderen Bereichen eine Bedeutung haben. So ist ein wirklich freier und zugleich schneller Internetzugang nicht nur ein wesentliches Erfolgskriterium für die Arbeit im Bereich Gaming. Es stellt vielmehr eine grundlegende Ressource für alle zukünftigen Bibliotheksaktivitäten dar.

Beginnen wir also mit der Analyse des Ist-Zustands. Um sich mit dem Thema Gaming zu beschäftigen, benötigen Sie eine Vielzahl an unterschiedlichen Ressourcen. Wenn Sie bestimmte Ressourcen nicht haben, bedeutet dies nicht, dass Sie daraus resultierend keine Aktivitäten im Bereich Gaming anbieten können. Es bedeutet vielleicht nur, dass bestimmte Aktivitäten für Sie nicht umsetzbar sind. Es kann auch bedeuten, dass wir uns nach alternativen Wegen umsehen müssen. Im Folgenden beschreibe ich die einzelnen Ressourcen und warum Sie sie benötigen. 


\section{Raum}

Wenn Sie in der Bibliothek Veranstaltungen zum Thema Gaming durchführen möchten, benötigen Sie dafür Raum. Je nachdem, welche und wie viele Gaming-Stationen Sie anbieten möchten, ergibt sich ein bestimmter Raumbedarf. Spiele, die auf der XBOX360 laufen und das Kinectsystem nutzen, benötigen sicherlich den meisten Platz. Dies liegt daran, dass das Kinect-System eine Gestensteuerung darstellt, d.h. der Körper des Spielers ist der Controller. Wenn also zwei Spieler spielen, benötigen sie den dafür notwendigen Platz. Bedenken Sie aber, dass Sie nicht nur Platz für die Spieler benötigen. Bei Events in der Bibliothek werden wir auch Zuschauer haben. Insofern brauchen wir auch dafür Platz. Die Zuschauer sollten ca. einen Meter Abstand zu den Spielern haben, damit das System sie nicht als Spieler identifiziert. Des Weiteren sollte ausreichend Platz zwischen den Gaming-Stationen vorhanden sein. Auch hier benötigt das Kinect-System den meisten Platz. Planen Sie mindestens zwei Meter Abstand ein. Ansonsten besteht die Gefahr, dass das System die Nachbarspieler ebenfalls identifiziert und es dadurch zu Störungen kommt. Wenn Sie nur mit klassischen Controllern - inkl. der Move der Playstation - arbeiten, reicht ein geringerer Platz aus. Aber auch hier sollten Sie auf jeden Fall ausreichend Platz für die Zuschauer einplanen. Stellen Sie auch Sitzgelegenheiten zur Verfügung. Nicht alle Teilnehmer und/ oder Zuschauer können die ganze Zeit stehen. Darüber hinaus sollten Sie auch Platz zum Ausruhen zur Verfügung stellen. Gaming kann anstrengend sein. Und es wäre gut, wenn Ihre Bibliothek nicht nur als Ort des Spielens angesehen wird. Schaffen Sie also eine Wohlfühlatmosphäre.

Unter Umständen können in der Bibliothek auch Bilder aus dem Bereich Gaming aufgehängt werden. Wenn Sie eine oder mehrere Stationen dauerhaft in der Bibliothek installieren möchten, benötigen Sie nicht nur den Platz für die Spieler und die Geräte. Sie sollten zudem überlegen, wie man die anderen Bibliothekskunden nicht stört. Immerhin ist für viele Bibliothekskunden die Bibliothek ein Ort der Ruhe, an dem sie sich zur Lektüre oder zum Lernen zurückziehen können. Wenig sinnvoll ist die Idee, das Spielen ohne Ton anzubieten, damit andere Bibliothekskunden nicht gestört werden.

Wie bereits besprochen geht es beim Gaming $\mathrm{u}$. a. um ein gut funktionierendes Feedback. Dieses Feedback wird in der Regel auf mehreren Kanälen gleichzeitig vermittelt und Töne, Musik, Sprache etc. gehören dazu. Nehmen Sie den Spielen den Ton weg, machen Sie sie letztlich kaputt. Zudem müssen Sie auch mit Geräuschen durch die Spieler rechnen. Die Spieler werden sich unterhalten. Gaming ist - wie gesagt - eine soziale Aktivität. Wenn Sie in Ihrer Bibliothek keinen Raum zur Verfügung stellen können, der den Anforderungen für eine dauerhafte Nutzung von Games ermöglicht, sollten Sie sich eher auf Events konzentrieren.

Leihen Sie sich eine XBOX360 inkl. Kinect, Monitor, Lautsprecher etc. aus und testen Sie in der Bibliothek die vorhandenen Örtlichkeiten.

Zeit

Es mag etwas verwundern, dass ich auch den Faktor Zeit benenne, jedoch ist er für eine erfolgreiche und nachhaltige Arbeit mit dem Thema unerlässlich. Viele Bibliotheksmitarbeiter machen den Fehler, dass sie den zeitlichen Aufwand unterschätzen. Sehr oft wird davon ausgegangen, dass man sich dem Thema auf die gleiche Art und Weise nähern kann, wie dies auch in anderen Bereichen getan wird. Aber Gaming ist 
anders. Selbst wenn Sie sich nur im Rahmen der Bestandsarbeit um das Thema Gaming kümmern möchten, benötigen Sie Zeit für die Recherche. Da Sie mit dem Thema wahrscheinlich nicht so vertraut sind, werden Sie Zeit brauchen, sich in die Thematik einzuarbeiten. Dies beginnt schon mit dem Erlernen bestimmter Fachbegriffe.

\section{Freier Internetzugang}

Ein weiteres wichtiges Kriterium ist ein freier Internetzugang. Sie benötigen diesen aus verschiedenen Gründen. Zum einen benötigen Konsolen, PCs, Tablet-PCs, Smartphones etc., also die gesamte Hardware, kontinuierliche Systemupdates. Wenn Sie diese Updates nicht bekommen, sind manche Spiele nicht spielbar. So benötigt z. B. die XBOX360 mit dem Kinect-System immer wieder Updates, um das Kinect-System $\mathrm{zu}$ verfeinern und damit die neuesten Spiele spielbar zu machen. Zum anderen benötigen Sie den Zugang, um Spiele downloaden zu können. Immer mehr Spiele oder auch nur neue Level und Updates werden gar nicht mehr auf einem physischen Datenträger angeboten. Diese Dateien müssen aus dem Internet heruntergeladen werden. Gleiches gilt für Spiele, die als Apps. auf Tablet-PCs oder Smartphones geladen werden. Unabhängig vom Zugang zu den Spielen und zu weiterer Software benötigen Sie den freien Internetzugang auch, um im Internet zum Thema Gaming recherchieren zu können. Nun mögen Sie einwenden, dass Sie so viel Zeit z. B. für aufwendige Recherchen gar nicht aufbringen möchten oder können. Aber selbst wenn Sie sich nicht weiter informieren möchten - was ich jedoch dringend empfehlen würde - werden Sie mit Sicherheit Informationen aus dem Netz zu bestimmten Fragestellungen benötigen, mit denen Sie arbeiten. Früher oder später werden Ihre Kunden mit Fragen zu bestimmten Spielen auf Sie zukommen. Natürlich müssen Sie kein Experte für jedes Spiel sein. Aber Sie sollten in der Lage sein, Ihren Kunden im Falle eines Falls eine Online-Plattform empfehlen können, auf der er wahrscheinlich die richtige Antwort findet. Ein weiterer wichtiger Punkt ist der Zugang zu den sozialen Netzwerken wie Facebook, Gaming-Communities, Gaming-Plattformen etc. Je nachdem, welche Zielgruppe Sie gerne erreichen möchten, müssen Sie deren Kommunikationswege nutzen. Bestimmte Zielgruppen mögen Sie vielleicht mit Aushängen, Flyern oder Anzeigen in den jeweiligen Zeitungen erreichen. Aber bei einigen Zielgruppen spielt sich der Informationsaustausch über Veranstaltungen und neue Angebote auf Plattformen wie Facebook und Co ab. Wie gesagt, wenn Sie keinen freien Internetzugang haben, heißt das nicht, dass eine Arbeit im Bereich Gaming nicht möglich ist. Wir müssen dann aber über Alternativen nachdenken oder aber auf bestimmte Konzepte verzichten.

Testen Sie nicht nur die Internetverbindung an sich. Testen Sie auch, ob die Geschwindigkeit der Verbindung ausreicht. Testen Sie unbedingt auch, ob diese sich mit den jeweiligen Netzwerken wie z. B. dem Playstation-Network verbinden können. In einigen Fällen kann die Bibliothek zwar auf das Internet, aber nicht auf die jeweiligen Networks zugreifen.

\section{Alternativen}

Wenn Sie keinen freien Internetzugang haben, kann man im ersten Schritt mit dem Träger und/oder der IT-Abteilung sprechen, um zumindest auf eine gewisse Anzahl an Online-Plattformen zugreifen zu können. Besonders wichtig ist in diesem Zusammenhang Youtube, da man hier sehr viele Tutorial-Videos zur Gaming-Hardware und natürlich zu den Games selbst finden kann. 


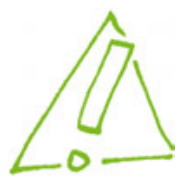

Legen Sie sich für jedes Spiel eine Linkliste an. Es reicht, wenn Sie drei Links pro Spiel finden. Am besten Sie suchen zuerst bei Youtube nach einem Tutorial. Dann sollten Sie versuchen, eine Quelle zu finden, auf der beschrieben wird, ob und wenn ja welche optionalen Zusatzaktivitäten im Spiel vorhanden sind. Sie können die Erstellung dieser Linkliste auch als eigenes Gaming-Projekt zusammen mit Gamern durchführen. Wichtig ist nur, dass Sie diese Liste nicht in der Bibliothek „verstecken“, sondern diese öffentlich - am besten online - zugänglich ist.

\section{Freie PCs}

Wenn Sie vorhaben, ein dauerhaftes lokal spielbares Angebot im Bereich PC-Games anzubieten, brauchen Sie dafür natürlich freie Rechner. Wenn man also in Ihrer Bibliothek an fest installierten Computern spielen darf, werden diese Rechner u.U. für andere Aktivitäten nicht mehr zur Verfügung stehen. Wenn Sie also nur ein oder zwei Internetarbeitsplätze anbieten können, die zudem auch für das Schreiben von Briefen etc. benutzt werden sollen, werden Sie hier nicht viel erreichen können.

\section{Eigener Server}

Für gewisse Spiele bzw. gewisse Aktivitäten benötigt man einen eigenen Server. Wenn Sie z. B. eine eigene Minecraft-Welt aufbauen möchten, geht dies nicht ohne eigenen Server. Natürlich können Sie auch bei verschiedenen Anbietern einen solchen Server mieten. Mit eigenem Server ist jedoch nicht nur die Hardware, sondern auch das notwendige Know-how im Umgang damit gemeint. Ein eigener Server ist jedoch nur bei ganz bestimmten Aktivitäten notwendig. Die meisten Bibliotheken werden keinen eigenen Server benötigen.

\section{Freie Geldmittel}

Ein besonders wichtiger Punkt ist die Frage, ob Sie über freie Geldmittel verfügen. In vielen Bibliotheken sind die meisten, wenn nicht alle Mittel zweckgebunden. Es wäre aber hilfreich, wenn Sie über ein kleines Budget selbst verfügen könnten. Dabei geht es natürlich u. a. um den Ankauf von Hard- und Software. Letzteres kann man sicherlich auch über die Nutzung des Budgets der Bestandsarbeit lösen. Es kann aber durchaus sein, dass Sie z. B. Mittel für neue Hardware benötigen. Des Weiteren werden Sie früher oder später auf externe Hilfe z. B. für Schulungen, Beratungs-Workshops, Events etc. zurückgreifen müssen. Dies ist vor allen dann relevant, wenn Sie sich längerfristig mit dem Thema beschäftigen möchten.

\section{Offenes Umfeld}

Ein sehr wichtiges Erfolgskriterium ist ein offenes Umfeld. So spannend und wichtig das Thema Gaming auch sein mag, es wird immer Menschen geben, die das Thema sehr kritisch sehen. In den letzten Jahren wurde das Thema in der breiten Öffentlichkeit immer wieder negativ beleuchtet. Themen wie Spielsucht, Gewaltdarstellungen etc. sorgen dafür, dass viele Menschen besorgt sind. Zudem wird Gaming gerne als sinnloser, unsozialer etc. Zeitvertreib angesehen. Sie müssen als Bibliothek „fühlbar“ dem Thema Gaming positiv gegenüber stehen. Ansonsten wird es nicht nur für Sie 
schwer. Gamer kommen nicht gerne an einen Ort, an dem sie spüren, dass ihre Kultur eigentlich nicht erwünscht ist.

\section{Netzwerk}

Gerade für kleine Bibliotheken ist es fast unmöglich, das Thema Gaming alleine zu behandeln. Aus diesem Grund ist ein verlässliches Netzwerk von großer Bedeutung. Dieses Netzwerk kann bestehen aus:

- anderen Bibliotheken, die sich auch mit dem Thema beschäftigen möchten,

- anderen Institutionen aus Ihrer Stadt/Gemeinde, die an dem Thema interessiert sind oder vielleicht sogar schon mit eigenen Aktivitäten begonnen haben. Dazu können z. B. gehören:

- Jugendamt

- Jugendclubs

- Schulen

- gemeinnützige Projekte

- Gamern bzw. Gaming-Communities,

- interessierten Eltern, Lehrern,

- dem Kulturamt,

- Unternehmen (z. B. als Sponsoren oder Kooperationspartner),

- Plattformen wie „games4culture“.

Laden Sie die Institutionen, Unternehmen und Einzelpersonen, die Interesse an dem Thema haben könnten, zu sich ein. Sie können auch in einem ersten Schritt einen Gaming-Stammtisch einrichten. Wenn Sie mögliche Kooperationspartner gefunden haben, können Sie dann ein kleines Gaming-Netzwerk aufbauen.

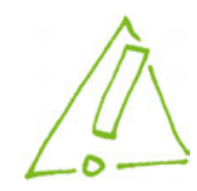

\section{Geräte}

Wie Sie bereits im Laufe des Buches erfahren konnten, brauchen Sie auf jeden Fall technische Geräte. Dazu gehören natürlich Gaming-PCs, Konsolen, Controller, Headsets, Lautsprecher, Akku-Ladegräte etc. Im Laufe der Jahre werden Sie diese Geräte austauschen oder reparieren müssen.

\section{Offene Kollegen}

Sie mögen ein richtiger Gaming-Maniac sein. Vielleicht sind Sie schon seit vielen, vielen Jahren aktiver Gamer. Vielleicht haben Sie Kinder, die jetzt in einem Alter sind, in dem sie selbst mit dem Spielen von Computerspielen beginnen. Auf jeden Fall möchten Sie das Thema zum Teil Ihrer Bibliotheksarbeit machen. Das ist großartig! Aber Sie werden nicht erfolgreich sein, wenn Sie keine Unterstützung von Ihren Kollegen bekommen. Damit meine ich nicht nur, dass Ihre Kollegen das Thema dulden bzw. ertragen. Es geht um aktive Mithilfe, wenn möglich von allen Kollegen. Ohne diese Unterstützung wird es sehr schwer werden. Das Problem ist nicht nur, dass Sie u. U. bei Ihrem Gaming-Event in der Bibliothek in Arbeit ertrinken. Denn was ist, wenn Sie erfolgreich sind? Was ist, wenn Ihre Angebote im Gaming-Bereich so gut funktionieren, dass Sie immer mehr Menschen damit anlocken? 
In vielen Fällen werden neue Aufgaben und Projekte folgendermaßen geplant: Man überlegt sich, welche Aufgaben jeder hat, damit man das Thema umsetzen kann. Dies wird in der Regel so geplant, dass man die Arbeit mit dem jetzigen Wissensstand umsetzen kann. Aber das kann problematisch werden. Es ist wichtig, dass Sie ihren Personaleinsatz bei einem Thema wie z. B. Gaming immer so planen, dass Sie keine Probleme bekommen, wenn Sie erfolgreich sind. Planen Sie also vor allem Events immer mit vielen Teilnehmern.

Unabhängig von der Frage, welche Aktivitäten Sie im Bereich Gaming wirklich umsetzen wollen, sollten Sie bereits zu diesem Zeitpunkt davon ausgehen, dass Sie alle genannten Ressourcen benötigen. Nun sollten Sie also analysieren, welche Ressourcen Ihnen zur Verfügung stehen und welche Ihnen zur Verfügung stehen könnten, wenn Sie sie benötigen.

Wenn Sie nun als Ergebnis herausfinden, dass Ihnen keine der genannten Ressourcen zur Verfügung stehen, ist dies nicht gleichbedeutend mit dem Ende aller Bemühungen im Bereich Gaming. Es bedeutet aber, dass manche Aktivitäten durch Sie nicht durchführbar sind und dass Sie zudem mehr Energie aufwenden müssen, um mit dem Thema Gaming zu starten.

\section{Gaming-SWOT-Analyse}

Ein sehr hilfreiches Tool ist die sogenannte SWOT-Analyse. Diese Analyse wird eigentlich im Marketing angewendet. Die vier Buchstaben stehen für Strengths, Weaknesses, Opportunities und Threats. Übersetzt ins Deutsche geht es um eine Analyse der Stärken, Schwächen, Chancen und Risiken, die sich aus der Arbeit mit einem bestimmten Thema ergeben. Eine SWOT-Analyse kann helfen, Ihre aktuelle Situation und Ihre Möglichkeiten und etwaige Problemstellungen zu analysieren. Wichtig ist dabei, dass Sie vorab alle relevanten Parameter definieren. Es geht also nicht nur um technische Ressourcen, sondern auch um die Haltung des Trägers bzw. des gesamten Umfeldes. Und auch ein Blick auf die demographische Lage Ihrer Stadt oder Gemeinde ist wichtig. Auch wenn Gaming kein reines Jugendthema ist, ist es hilfreich zu wissen, für welche Zielgruppe Sie das Thema bearbeiten möchten. Eine SWOT-Analyse funktioniert aber nur, wenn Sie ehrlich umgesetzt wird, d. h. versuchen Sie nicht, Ihre Situation schön zu reden. Und wenn Sie am Ende einer SWOT-Analyse feststellen, dass Sie eigentlich nicht in der Lage sind, eine wirklich spannende und nachhaltige Gaming-Bibliothek zu werden, ist dies noch nicht das Ende. Es bedeutet letztlich nur, dass Sie etwas länger brauchen werden.

\section{Kleine und große Bibliotheken}

Ich weiß, dass manche Bibliotheksmitarbeiter der Meinung sind, man müsse eine besonders große Bibliothek sein, um im Bereich Gaming erfolgreich sein zu können. Aber dem ist nicht so oder anders ausgedrückt: Es ist keine Frage der Größe einer Bibliothek, ob sie im Bereich Gaming erfolgreich sein kann oder nicht. Natürlich verfügt eine große Bibliothek über mehr Ressourcen, aber manchmal kann diese Menge an Ressourcen auch hinderlich sein. Manche große Bibliothek hat im Laufe der Zeit Strukturen aufgebaut, die es ihr nicht mehr möglich machen, flexibel zu agieren. Wenn Sie also in einer kleinen Bibliothek arbeiten, können Sie genauso eine GamingBibliothek werden wie jede andere Einrichtung auch. Der alles entscheidende Faktor ist der Wille. Entweder Sie wollen im Bereich Gaming aktiv sein oder nicht. 
Wenn Sie die Ressourcenanalyse abgeschlossen haben, sollten Sie nun analysieren, was Sie im Bereich Gaming schon alles tun. Nehmen Sie sich am besten die letzten drei Jahre vor und überlegen Sie, was Sie erreicht haben und was nicht. Analysieren Sie dabei auch, warum Sie mit dem Thema gestartet sind und welche Ziele Sie dabei verfolgten. Versuchen Sie dann ebenfalls zu überlegen, ob sich nach der Lektüre dieses Buches bei Ihnen hinsichtlich der Zielsetzungen etc. etwas geändert hat. Stellen Sie sich folgende Fragen:

1. Welche Ziele verfolgt meine Bibliothek im Bereich Gaming?

2. Welche Zielgruppe wollen wir erreichen?

3. Warum wollen wir diese Zielgruppe erreichen?

4. Wie viele Mitarbeiter haben bereits Erfahrungen im Bereich Gaming?

5. Betreibt die Bibliothek ein aktives Bestandsmanagement im Bereich Gaming?

6. Welche Medien hat die Bibliothek außerhalb der eigentlichen Games im Bestand? (dazu gehören z. B. Fachbücher, Magazine etc.)

7. Wie reagiert das Team und wie das Umfeld auf das Thema Gaming?

Diese Fragen sollten Sie am besten in Ihrem Team besprechen. Je öfter Sie die aktuelle Situation analysieren, desto schneller wissen Sie, wo Ihre Stärken und wo Ihre Schwächen liegen.

Wenn Sie Ihre Ziele und Zielgruppen analysieren, sollten Sie darauf achten, dass Ihre Ziele und Zielgruppendefinitionen an folgende Parameter angepasst werden:

1. Gaming ist kein alleiniges Jugendthema.

2. Gaming ist nicht dafür da, um Menschen in die Bibliothek zu locken, damit man sie dann zum Lesen von Büchern animiert.

3. Ein Bestand im Bereich Gaming ist ein guter Start, er reicht aber nicht aus.

Legen Sie nun erneut Ihre Ziele und Ihre Zielgruppen fest. Im Anschluss daran sollten Sie überlegen, inwieweit auch andere Aktivitäten Ihrer Bibliothek diese Ziele erreichen. Ebenso sollten Sie überlegen, ob Sie die festgelegte Zielgruppe bzw. die festgelegten Zielgruppen schon heute, sei es mit den vorhandenen Gaming-Aktivitäten oder auch mit anderen Aktivitäten, erreichen oder nicht. Wenn Sie dabei herausfinden, dass Sie einige Teile der definierten Zielgruppe nicht erreichen, sollten Sie überlegen, woran das liegen könnte. Haben Sie vielleicht keine interessanten Angebote für diese Zielgruppe? Erreicht Ihre Kommunikationsstrategie diese Zielgruppe nicht?

Schließlich sollten Sie überlegen, inwieweit Gaming zu anderen bereits vorhandenen Aktivitäten Ihrer Bibliothek passen könnte. Nehmen wir als Beispiel die verschiedenen Informationsangebote. Auch für das Thema Gaming brauchen wir erfolgreiche Informationsangebote und es wäre einfacher, wenn wir vorhandene Aktivitäten integrieren könnten.

\section{Los geht's}

Nun möchten Sie sicherlich endlich loslegen und ja, es gibt nun einiges zu tun. Das Wichtigste ist jetzt, dass Sie sich selbst weiterbilden. Recherchieren Sie im Internet, besuchen die Workshops, besuchen Sie Gaming-Events, lesen Sie Fachbücher und vor allem: Spielen Sie so viele Spiele wie möglich. Ich weiß, es mag sich etwas komisch anfühlen, aber wenn Sie nicht spielen, können Sie Spielen nicht verstehen und damit verbunden auch keine sinnvollen Services anbieten. Stellen Sie sich ein- 
fach vor, Sie würden in einer Bibliothek arbeiten und hätten noch nie ein Buch gelesen oder vielleicht nur ein- oder zweimal einen Blick in ein Buch geworfen: Würden Sie sich dann zutrauen, Bibliotheksmitarbeiter zu sein? Sie müssen nicht alle Genres durchspielen, aber Sie müssen spielen. Am besten wäre es, wenn Sie dies im Team machen. Sie können auch externe Anbieter bitten, einen Workshop bei Ihnen durchzuführen. Und Spielen meint nicht, einen Tag etwas zu probieren. Sie müssen kontinuierlich Spiele ausprobieren. Es geht nicht darum, dass Sie Ihre ganze Arbeitszeit dafür investieren. Aber es sollte zumindest für einen gewissen Zeitraum ein wichtiges Thema sein. Besuchen Sie auch Foren und Communities und lernen Sie von den Gamern. Am besten nehmen Sie sich jeden Tag 20 Minuten für das Thema Zeit. Halbtags- oder Stundenkräfte entsprechend weniger. Jetzt werden Sie einwenden, dass das ganz schön viel Zeit ist. Ja, das ist es. Und je länger Sie warten, umso mehr Zeit werden Sie brauchen.

Wenn Sie die Analysen abgeschlossen haben und zudem fleißig gespielt haben, geht es nun darum, festzulegen, mit welchen Services Sie beginnen möchten. Behandeln Sie in diesem Moment Ihre Bestandsarbeit ebenfalls als neuen Service. Sie beginnen am besten mit der Frage, wie Sie in Zukunft das Thema Bestandsarbeit angehen möchten. Ich habe dazu ja schon einiges geschrieben, deshalb hier nur nochmal der Hinweis, darauf zu achten, dass Sie Spiele aus den drei Kategorien „aktuelle Spiele“, „Kunstspiele und Dauerbrenner“ sowie „Retro-Games“ anbieten bzw. im Bestand haben. Versuchen Sie bei der zweiten und dritten Kategorie einen Erwerbungsplan für ein Jahr zu erstellen. Diesen sollten Sie mit anderen Bibliotheken besprechen. Sie können auch überlegen, ob Sie einen Gaming-Verbund aufbauen, bei dem Sie Spiele untereinander austauschen oder aber in den Gaming-Aktivitäten kooperieren. Dies eignet sich besonders für kleine Bibliotheken, die nicht über die Ressourcen verfügen, um das Thema alleine zu bearbeiten.

Danach kümmern Sie sich um die möglichen Events und Projekte. Beginnen Sie unbedingt mit etwas Einfachem. Veranstalten Sie einen kleinen Gaming-Event mit nur einer Konsole. Vielleicht veranstalten Sie einen eintägigen internen GamingWorkshop, bei dem Sie und Ihr Team erfahren, um was es beim Thema Gaming geht und bei dem Sie ein paar Spiele und Konsolen ausprobieren können. Fahren Sie zur Gamescom. Alle diese kleinen Dinge können Sie ohne großen Aufwand umsetzen. Sie werden dabei einiges lernen und viele interessante Menschen kennenlernen. Sorgen Sie dafür, dass die Belastung des Teams nicht zu groß wird.

Schließlich kümmern Sie sich um die Weiterentwicklung der Informationsdienste. Sorgen Sie dafür, dass Ihre Bibliothek zu jedem Spiel auch ein Let's Play-Video kennt und dieses auch kommunizieren kann. Dies ist nur ein kleiner Schritt, aber er bedeutet eine massive Verbesserung Ihres Service-Angebots.

Schließlich überlegen Sie, wie Sie die Gaming-Kultur in Ihre eigene Institutionskultur integrieren können.

Was Ihnen grundsätzlich klar sein muss, ist Folgendes: Die Welt des Gamings ist sehr groß und sie funktioniert bis jetzt wunderbar ohne Bibliotheken. Diese Welt - dies ist letztlich auch beim Internet, dem mobilen Internet, Social Media etc. der Fall - funktioniert wunderbar ohne Bibliotheken. Das bedeutet, Sie fangen von vorne an. Bibliotheken können auf der anderen Seite eine ganz große Rolle in der GamingKultur spielen. Wenn Sie verstehen, dass es um eine andere Bibliothek geht. 


\section{Die Bibliothek als Gaming-Ort}

Ich habe immer wieder auf den Bereich der Bibliothek als Gaming-Ort hingewiesen. Nun möchte ich nochmal zusammenfassend beschreiben, was damit gemeint ist. In den letzten Jahren hat man verschiedene Definitionen für Bibliotheken entwickelt. Die Bibliothek ist demnach ein Lernort, ein kultureller Ort, ein sozialer Ort, ein RuheOrt, ein nichtkommerzieller Ort, ein offener Ort etc. Diese Begriffe bedeuten nichts anderes, als dass man der Bibliothek verschiedene Funktionen zuordnet, die sie dann erfüllen soll. Nun könnte ich es mir einfach machen und sagen, dass Gaming in allen diesen Funktionen vorhanden ist und es deshalb keiner neuen Definitionen und Funktionen bedarf. Ich bin jedoch der Meinung, dass wir genau eine solche Definition brauchen. Deshalb spreche ich von der Bibliothek als Gaming-Ort. Diese Funktion oder Definition ist nicht als Konkurrenz zu anderen Modellen gedacht. Sie steht für ein Ziel, ein Modell und sie steht gleichzeitig für eine neue Denk- und Arbeitsweise. Was also genau ist die Bibliothek als Gaming-Ort?

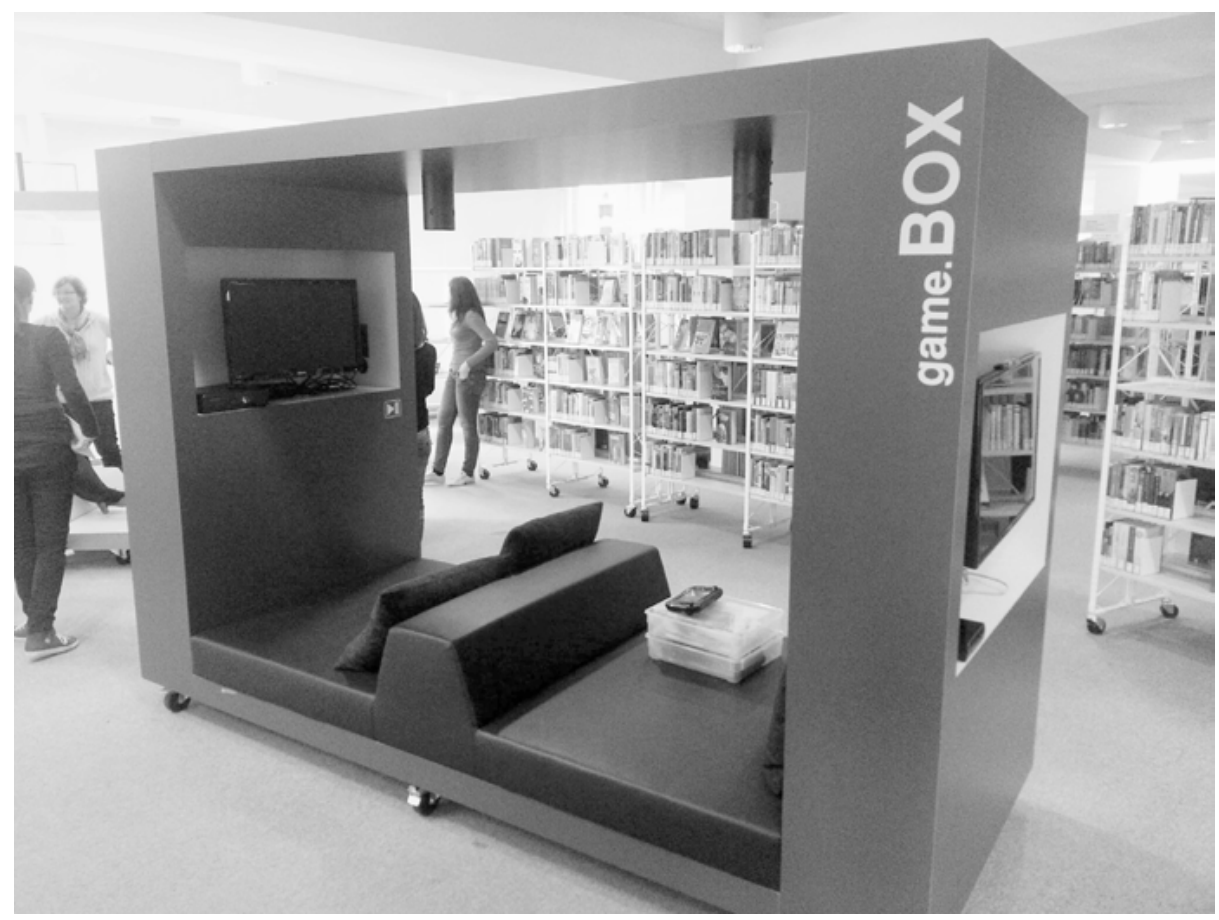

Abb. 26 Gaming-Station in der Stadtbibliothek Minden

1. Sie ist ein Ort, an dem Games die gleiche Bedeutung wie Bücher haben.

2. Sie ist der Ort, an dem Gamer und die Gaming-Kultur willkommen sind.

3. Sie ist der Ort, an dem u. a. mit Games gelernt wird.

4. Sie ist der Ort, an dem der spielerische Umgang mit Inhalten und Medien möglich ist.

5. Sie ist der Ort, an dem man alles ausprobieren kann und man Fehler machen darf.

6. Sie ist der Ort, an dem Spaß beim Lernen, Arbeiten oder bei Freizeitaktivitäten im Vordergrund steht.

7. Sie ist der Ort, der von den Nutzern/Kunden/Gamern gestaltet wird und nicht von den Bibliotheksmitarbeitern.

8. Sie versteht sich als offene Plattform, die die Menschen nutzen können, für was auch immer sie sie nutzen möchten. 
9. Sie ist der Ort der Vernetzung und sie funktioniert wie ein Netzwerk.

10. Sie ist der Ort, an dem sich Bibliotheksmitarbeiter als Teil einer viel größeren Community verstehen.

11. Sie ist der Ort, an dem neue analoge und digitale Welten erschaffen werden.

12. Sie ist der Ort der Quests und Abenteuer.

13. Sie ist der Ort, an dem man sich aber auch zurückziehen kann, um innezuhalten und zu reflektieren.

Diese dreizehn Punkte beschreiben für mich die Gaming-Bibliothek als Ort. Und dies sollte das gedankliche Ziel Ihrer Arbeit sein. Das bedeutet, dass Sie sich nicht nur darüber Gedanken machen sollten, welche Spiele und Bücher und Magazine Sie in den Bestand aufnehmen. Es reicht auch nicht aus, ein paar Veranstaltungen zu entwickeln und zu realisieren. Die Bibliothek als Ganzes wird verändert, wird besser, wenn Sie Gaming und die damit verbundenen Denk- und Arbeitsweisen in Ihre Arbeit integrieren.

\section{Die vier Tätigkeitsfelder der ultimativen Gaming-Bibliothek}

Wenn wir uns überlegen, was eine Bibliothek im Bereich Gaming tun kann, dann wird sich in den meisten Fällen auf die Bereiche Bestand und Events fokussiert. Natürlich wäre es ein großer Erfolg, wenn alle Bibliotheken in diesen beiden Bereichen aktiv wären. Aber wenn man wirklich etwas mit Games machen möchte bzw. zur ultimativen Gaming-Bibliothek werden möchte, dann gibt es letztlich vier Tätigkeitsfelder, in denen Bibliotheken im Bereich Gaming aktiv sein sollten:

1. Bestandsarbeit: Hierzu gehört der Aufbau eines Bestandes an Games (in den drei beschriebenen Bereichen), Büchern, Magazinen, Videos etc.

2. Veranstaltungen im Bereich Gaming: Hierzu gehören alle beschriebenen Veranstaltungen, also z. B. Gaming-Wettbewerbe, Storytelling-Projekte, Bibcraft, Case-Modding etc.

3. Neue Informationsdienste: Dazu gehört, dass Sie in der Lage sind, auch beim Thema Gaming wertvolle Informationen anzubieten. Dies können Informationen für Gamer oder aber für deren Umfeld wie z.B. Lehrer oder Eltern sein. Ebenfalls in diesen Bereich gehört die Arbeit mit Youtube-Videos wie Let's Play etc.

4. Integration des Thema in alle anderen Tätigkeitsfelder der Bibliothek: Hierzu gehört die Nutzung von Gaming-Elementen in anderen Bibliotheksangeboten sowie die Fokussierung auf die unter „die Bibliothek als Gaming-Ort“ benannten Punkte.

Nutzen Sie unbedingt die Plattform www.mobygames.com. Es handelt sich um eine riesige und umfassende Datenbank mit Informationen zu nahezu allen Games, die es jemals gegeben hat.

Was Sie nun tun sollten, ist, Ihre gesamten Aktivitäten dahingegen zu analysieren, in welches dieser vier Felder sie jeweils gehören. Erstellen Sie eine Matrix mit den vier Feldern und tragen Sie alle Ihre vorhandenen Aktivitäten in das jeweilige Feld ein.

Wenn Sie diese Analyse abgeschlossen haben, liegen Ihnen viele wichtige Informationen zu Ihrer aktuellen Situation vor. Sie wissen nun, in welchen Bereichen 
Sie schon aktiv sind und wo nicht. Sie wissen, welche Ressourcen Sie benötigen und welche Ressourcen Sie davon schon haben. Sie wissen ebenfalls, welche Veranstaltungen interessant sind bzw. welche Sie umsetzen können. Und Sie wissen, was Ihre Mitarbeiter im Bereich Gaming an Erfahrungen haben.

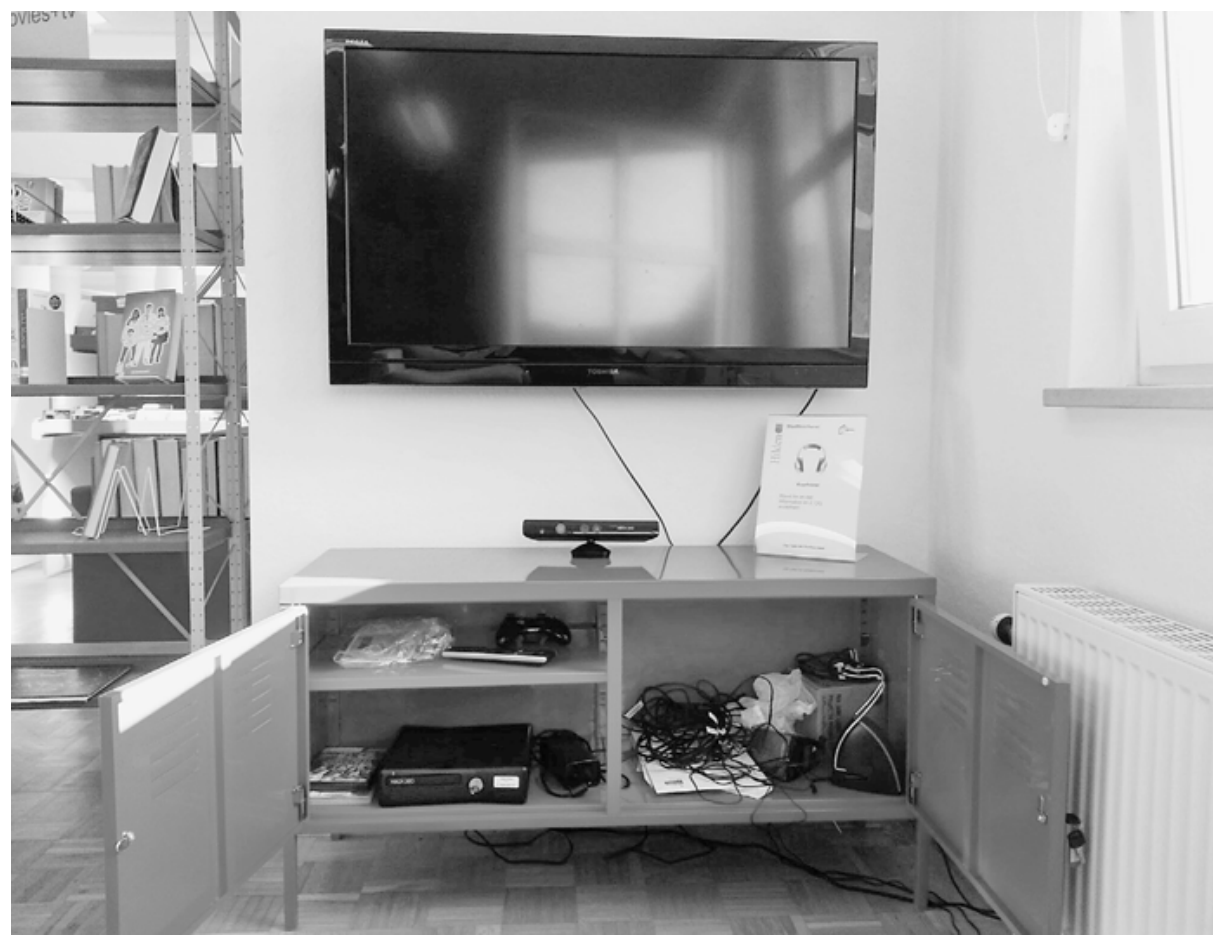

Abb. 27 Gamingstation in der Stadtbücherei Hilden

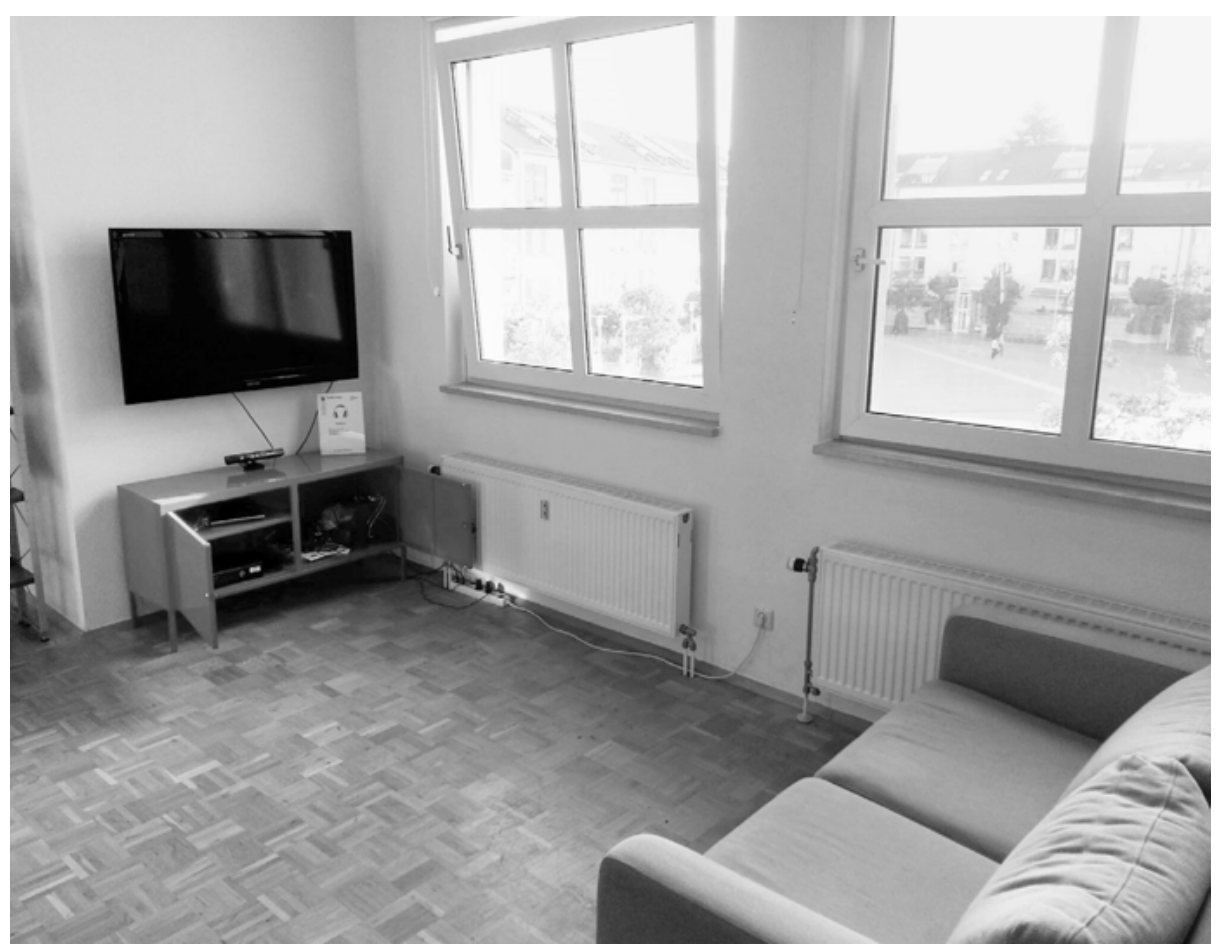

Abb. 28 Gamingstation in der Stadtbücherei Hilden 
Aus diesen genannten Punkten erstellen Sie nun eine Liste von Dingen, die Ihre Bibliothek umsetzen muss, um eine echte Gaming-Bibliothek zu werden. Dabei gilt aber Folgendes zu beachten:

Wenn Sie Ihre aktuelle Situation analysiert haben, kann es sein, dass das Ergebnis im Vergleich zu dem, was ich bis jetzt in diesem Buch an Ideen und Zielsetzungen geschrieben habe, sehr traurig aussieht. Dies wird mit Sicherheit nicht Ihr Fehler sein. In den allermeisten Fällen liegt dies vielmehr an Ihrer Gesamtsituation. So erlebe ich sehr oft, dass schlichtweg die Ressourcen fehlen und/oder das Umfeld bzw. der Träger der Bibliothek das Thema Gaming ablehnen. In vielen Fällen ist es auch so, dass das Team kein großes Interesse an diesem Thema hat und Sie nun als Einzelkämpfer nicht nur die Gaming-Aktivitäten umsetzen sollen, sondern sich zudem auch noch vor Ihren Kollegen rechtfertigen müssen, warum Sie sich jetzt mit Games beschäftigen, wo doch gerade so viel andere Arbeit ansteht. Lassen Sie sich also von der Analyse nicht demotivieren. Wir haben alle klein angefangen.

Gleichzeitig kann es sein, dass Sie das Gefühl haben, alle diese Punkte nicht erreichen zu können. Auch hier sollten Sie sich nicht verrückt machen lassen. Eine Gaming-Bibliothek wird man nicht, weil man einen besonders großen Gaming-Bestand hat und jeden Tag 20 Gaming-Events durchführt. Sie werden eine Gaming-Bibliothek, wenn Sie die 13 Punkte beherzigen und sich daran halten.

Wenn Sie nun die Analyse abgeschlossen haben, entwickeln Sie einen Plan. Und jetzt wird es spannend. Wie ich ja bereits erläutert habe, müssen Sie als Bibliothek selbst die Denk- und Arbeitsweisen der Gaming-Welt verstehen und in Ihre Arbeit integrieren. Und deshalb schlage ich vor, dass wir den Weg zu einer Gaming-Bibliothek, also alle Ihre nächsten Schritte, zu einem Game machen. Genauer gesagt nutzen wir bestimmte Gaming-Elemente, um die anstehenden Aufgaben zu lösen.

Als erstes müssen Sie noch einmal das Ziel definieren. Schreiben Sie sich genau auf, was Sie erreichen wollen. Das Ergebnis ist dann das, was Sie als Gaming-Bibliothek definieren. Dazu sollten aus allen vier Feldern bzw. Tätigkeitsfeldern Elemente vertreten sein. Als nächstes müssen Sie ganz genau aufschreiben, welche kleinen Schritte nötig sind, um das Ziel zu erreichen. Hier ist wichtig, dass Sie diese Schritte in ganz kleine Aufgaben unterteilen. Wenn es also darum geht, ein Gespräch mit dem Träger zu führen, dann bedeutet dies, Sie müssen überlegen, um was es in dem Gespräch gehen soll, Sie müssen einen Termin vereinbaren, Sie müssen das Gespräch vorbereiten, Sie müssen das Gespräch führen und Sie müssen es dann nacharbeiten. Es sind also viele kleine Aufgaben, die Sie erfüllen müssen. Und alle diese Aufgaben müssen Sie einmal definieren. Dazu gehört auch, dass Sie das eigene individuelle Fortkommen der Mitarbeiter definieren müssen. Wie bereits erwähnt müssen eine Vielzahl an Dingen recherchiert und erlernt werden. Dazu gehört auch das Ausprobieren von Spielen und der Hardware. Auch hier müssen Sie definieren, was genau getan werden muss.

Im Ergebnis haben Sie nun eine Liste mit Aufgaben und einer Aufteilung auf alle Mitarbeiter. Es gibt dabei Aufgaben, die man für sich macht wie z.B. das Ausprobieren eines Spiels, und Aufgaben, die Sie lösen müssen, um dem ganzen Team zu helfen. Das sind z.B. die Auswahl von Spielen und die Vorbereitung und Durchführung von Events. Und nun integrieren wir ein paar Gaming-Elemente in dieses Konstrukt.

Zuerst nehmen wir das Ziel. Dieses Ziel bekommt nun einen epochalen Namen. Nennen wir es z.B. „Gaming-Bib-Quest“. Dieses Ziel wollen wir erreichen. Und nun schreiben Sie auf, was dieses Ziel bedeutet, also was hat Ihre Bibliothek im Bereich Gaming an Aktivitäten umgesetzt, wenn das Ziel erreicht ist. Im nächsten Schritt versuchen Sie, die Schritte, die Sie vorab im Detail beschrieben haben, in Abschnitte einzuteilen. Und diese Abschnitte werden nun zu aufeinander aufbauenden Level. 
Jedes Level beschreibt also Aktivitäten, die Sie alleine oder im Team durchführen müssen. Wir bauen also einen spielerischen Projektplan. Dasselbe Modell nutzen wir auch für einen individuellen Plan für jeden Mitarbeiter. Sprechen diese individuellen Ziele unbedingt miteinander ab und akzeptieren Sie, dass es Mitarbeiter gibt, die mit dem Thema gar nichts anfangen können bzw. die sich total dagegen sträuben. Aber auch wenn wir natürlich Rücksicht nehmen, gibt es ein paar Grundelemente, die alle Mitarbeiter können sollten. Dies sind folgende Fähigkeiten:

1. Jeder Mitarbeiter sollte einmal ein Spiel ausprobiert haben.

2. Jeder Mitarbeiter sollte wissen, wie der gesamte Gaming-Bestand aussieht - inkl. der Bücher, Magazine etc.

3. Jeder Mitarbeiter sollte mit bestimmten Grundbegriffen vertraut sein.

4. Jeder Mitarbeiter sollte in der Lage sein, einem Kunden zu einem geliehenen Spiel oder einem Fachbuch ein passendes Youtube-Video zu empfehlen. Dazu gehört Let‘s Play, aber ebenso Videos von Vorträgen zu den Themen, welche in den Büchern behandelt werden.

5. Jeder Mitarbeiter weiß mit den Altersfreigaben der USK umzugehen und kann diese den Kunden erklären.

6. Jeder Mitarbeiter sollte einmal im Monat ein Gaming-Magazin lesen - zumindest in der Anfangsphase.

Diese sechs Fähigkeiten können Sie von jedem Mitarbeiter verlangen. Es sind die gleichen Fähigkeiten, die man auch bei allen anderen Medien braucht, um halbwegs seriös arbeiten zu können.

Unabhängig davon wäre es sinnvoll, wenn jeder Mitarbeiter mindestens einmal im Leben die Gamescom besucht. Ich weiß, das mag abgedroschen klingen, aber es würde vieles einfacher machen. Sie müssen wirklich nicht jeden Bibliothekartag besuchen. Sie können stattdessen auch mal etwas anderes machen - und vielleicht ein bisschen mehr Entertainment erleben. Und zur Buchmesse kommen ja auch viele Bibliotheksmitarbeiter :-)

Im Ergebnis haben Sie nun ein Ziel, dass Sie gemeinsam als Bibliothek erreichen wollen, um eine echte Gaming-Bibliothek zu werden. Sie haben zudem einen detaillierten Plan als Übersicht, der beschreibt, was für die Erreichung des Ziels getan werden muss. Sie wissen ebenso, was jeder Einzelne tun muss, um selbst dem Team helfen zu können. All diese Schritte werden nun in acht bis zehn Level unterteilt. Und nun beginnt das Spiel. Jede erfüllte Aufgabe bringt Punkte. Dies betrifft sowohl die Aufgaben, die das ganze Team erfüllen muss, als auch die individuellen Aufgaben. Allerdings sind die individuellen Aufgaben an die des Teams gekoppelt. Das bedeutet, dass die Bibliothek jedes Level zusammen abschließen muss. Jeder Mitarbeiter muss also sowohl seine individuellen als auch seinen Anteil an den Teamaufgaben erfüllen. Wenn man seine individuellen Aufgaben erfüllt hat, muss man also erst dem Team bei den Teamaufgaben helfen. Wenn auch dies abgeschlossen worden ist, aber einige Kollegen ihre eigenen individuellen Aufgaben noch nicht erfüllen konnten, dann muss man den Kollegen helfen. Erst wenn das Level komplett abgeschlossen ist, kann man gemeinsam das nächste Level angehen. Auf diese Art und Weise nehmen Sie alle Mitarbeiter mit. Da die Inhalte bzw. Aufgaben in den Level durch das Team besprochen wurden, besteht auch nicht die Gefahr der Überlastung.

Wie Sie das Ziel definieren und wie Sie die einzelnen Level definieren, bleibt Ihnen selbst überlassen. Dies müssen Sie immer individuell entscheiden. Für jede erfolgreich abgeschlossene Aufgabe bekommt das Team oder aber die Einzelperson Punkte. Achten Sie darauf, dass alle Personen immer die gleiche Punktzahl erreichen können. 
Auf diese Art und Weise wird der Fortschritt vergleichbar. Es geht dabei aber nicht um einen Wettbewerb Mitarbeiter gegen Mitarbeiter. Aber es entsteht so ein Feedbacksystem, das Ihnen als Team und als Einzelperson anzeigt, wo Sie gerade stehen.

Ganz wichtig: Feiern Sie Ihre Erfolge! Jedes erfolgreich abgeschlossene Level ist ein großer Erfolg. Gewöhnen Sie sich an, Erfolge zu feiern. Sie haben es sich verdient. Das Mindeste, das Sie tun müssen, ist Kaffee und Kuchen zu essen. Oder Sie öffnen für jedes erfolgreich abgeschlossene Level eine Flasche Champagner. In so vielen Bibliotheken arbeiten so tolle Menschen, aber leider wird immer vergessen, sich zu loben und sich zu belohnen. Aber genau das müssen Sie tun. Und auch das, die Belohnung, das Feedback, der Sieg, die Freude, all das sind zentrale Elemente der Gaming-Kultur.

Wenn Sie sich entscheiden, diesen Weg zu gehen, dann können Sie sogar noch einen drauflegen. Machen Sie daraus einen Wettbewerb! Treten Sie gegen andere Bibliotheken an. Dafür müssen nur die Anzahl der Level und Aufgaben angeglichen werden. Und dann geht's los. Sie können sich auf der Suche nach einer GegnerBibliothek auch gerne an mich wenden. Unter www.games4culture.de werde ich immer wieder einen diesbezüglichen Wettbewerb starten. Dabei werden wir nur die jeweiligen Bibliotheken miteinander vernetzen.

\section{Gaming-Bibliotheks-Netzwerke}

Selbst wenn Sie alle von mir genannten Punkte beherzigen und zu einer echten Gaming-Bibliothek werden, fehlt noch ein ganz wichtiges Element: ein Gaming-Bibliotheks-Netzwerk. Egal, wie mächtig und groß Ihre Bibliothek im Bereich Gaming geworden ist, im Vergleich zu den ansonsten vorhandenen Aktivitäten bleibt Ihre Bibliothek zwar ein Leuchtturm in der Dunkelheit, aber um wirklich ganz groß im Bereich Gaming zu sein, brauchen wir 800 Bibliotheken, die vernetzt als Gaming-Bibliotheken aktiv sind. Deshalb ist es wichtig, dass Sie wann immer möglich versuchen, andere Bibliotheken zum Mitmachen zu bewegen. Und einige der in diesem Buch beschriebenen Projekte wie z. B. Multiplayer-Events lassen sich nur in einem Netzwerk wirklich erfolgreich durchführen. Aber es geht auch darum, dass Sie voneinander lernen. In einem Netzwerk geht es darum, dass Sie aktiv Ihr Wissen teilen. Also auch dann, wenn Sie gar nicht danach gefragt werden. Viele Fragestellungen in Bibliotheken im Bereich Gaming sind auch in anderen Bibliotheken ein Thema und es gibt bei den weitaus meisten Problemen immer irgendwo eine Bibliothek, die dieses Problem bereits erfolgreich lösen konnte. Sie sparen mit einem Gaming-Bibliotheks-Netzwerk jede Menge Zeit und Geld. Eine Möglichkeit der Vernetzung bietet die Facebook-Gruppe „games4culture“. Wenn Sie allerdings keinen Account bei Facebook haben möchten, dann können Sie Ihre Fragen und Ideen auch auf anderen Plattformen stellen.

Der dritte Grund für ein Bibliotheksnetzwerk ist, dass Sie auf diese Art und Weise lernen, wie sich Gamer vernetzen. Wenn Sie Teil der Gaming-Kultur werden möchten, dann müssen Sie auch Teil der Gaming-Communities werden.

\section{Gaming-Bibliothek und Social Media}

Kommen wir nun zu einem weiteren wichtigen Thema: Gaming, Bibliotheken und Social Media. Dieses Buch ist ein Gaming-Buch. Deshalb werde ich jetzt nicht auf 100 Seiten über das Thema Social Media schreiben. Vielleicht schreibe ich darüber in meinem nächsten Buch. Aber Sie können Gaming nicht ohne Social Media angehen. 
Und dies hat verschiedene Gründe. Zum einen ist Social Media natürlich ein Element der Gaming-Kommunikation. Gamer haben sehr oft eine hohe Affinität zu modernen Kommunikations- und Medientechnologien. Zudem werden die klassischen Kommunikationskanäle von Bibliotheken nur von einer bestimmten Zielgruppe wahrgenommen. Meines Erachtens wird es für eine Bibliothek eine große Herausforderung werden, ohne weitreichende Social Media-Aktivitäten langfristig im Bereich Gaming erfolgreich zu sein. Denn es wird immer schwerer werden, Ihre Zielgruppe zu erreichen. Zum anderen brauchen Sie Social Media, um Teil der bereits vorhandenen Gaming-Communities zu werden. Die Kommunikation der Gamer untereinander findet immer mehr in sozialen Netzwerken statt. Man könnte auch sagen, Gaming und Social Media sind die beiden Seiten der gleichen Münze. Gaming und Social Media sind wie Bruder und Schwester. Die Denk- und Arbeitsweisen beider Welten sind ähnlich. Längst findet Gaming auch auf Plattformen wie Facebook statt. Genauer würde es ohne Gaming auch nicht diesen Erfolg von Facebook geben. Gleichzeitig wird Social Media ein Teil der Gamingwelt. Ein schönes Beispiel ist Tweetcraft. Es handelt sich dabei um ein kleines Tool, welches es dem Gamer ermöglicht, in der Welt von „World of Warcraft“ Twitter zu empfangen bzw. aus dem Spiel Tweets zu senden. Es ist nur ein kleines Beispiel von vielen, aber es zeigt, dass wir es längst mit einem Verschmelzungsprozess zwischen Gaming und Social Media zu tun haben.

Konkret bedeutet dies, Sie brauchen Social Media um Folgendes zu tun:

1. Kommunikation mit möglichen neuen Zielgruppen.

2. Die Bibliothek als Teil von Gaming-Communities.

3. Gaming-Communities als Teil der Bibliothek.

4. Aufbau von Know-how im Bereich Social-Gaming.

Unabhängig davon, dass jede Bibliothek umfassende Social Media-Aktivitäten aufbauen sollte, werden Sie im Bereich Gaming auf Dauer ohne Social Media nicht erfolgreich sein können. Natürlich können Sie auch ohne das Internet einen kleinen lokalen Gaming-Event veranstalten und diesen mit Flyern und Plakaten bewerben. Aber um eine richtige Gaming-Bibliothek zu sein, bedarf es weitaus mehr.

Zum jetzigen Zeitpunkt sind Aktivitäten auf Facebook, Twitter, Youtube, Vimeo und natürlich im Bereich Blogs unerlässlich. Selbst wenn Sie auf den genannten Plattformen am Anfang noch nicht selber aktiv sind, brauchen Sie diese, um sich zu informieren. Sie sollten also zumindest einen Account haben und wissen, wie man die Plattformen rudimentär bedient. Verstehen Sie Gaming unbedingt als Teil der digitalen Welt. Denken Sie immer daran, dass Sie Teil der Lebensrealität der Gamer werden wollen - nicht umgekehrt.

Sie können Social Media aktiv und passiv nutzen. Die aktive Nutzung meint das Arbeiten mit einer eigenen Facebook-Seite, einem eigenen Blog, einem eignen Twitter-Account etc. Man kann entweder das Thema auf den bereits vorhandenen Plattformen integrieren oder aber ein eigenes Angebot für den Bereich Gaming aufbauen. Bei kleinen Bibliotheken kann es auch sinnvoll sein, sich mit anderen Bibliotheken aus der Umgebung zusammenzutun. So entsteht dann z. B. ein lokales bzw. regionales Social Media-Angebot für das Thema Gaming. Diese Herangehensweise eignet sich auch für Bibliotheken in stark ländlichen Gebieten. Zur aktiven Nutzung gehört auch die Arbeit in Gaming-Communities. Hier können Sie nach Gamern in Ihrer Region suchen, Fragen stellen und auf die eigenen Aktivitäten aufmerksam machen.

Achten Sie unbedingt darauf, dass Sie nicht nur auf Facebook aktiv sind. Das Thema Gaming ist nicht ein reines Jugendthema und es gibt immer noch eine Vielzahl 
an Menschen, die keinen eigenen Account auf Facebook haben möchten. Deshalb lohnt es sich auf jeden Fall, auch einen Blog anzubieten.

Die passive Nutzung meint das Scannen verschiedener Social Media-Angebote. Am wichtigsten ist die Nutzung eines RSS-Readers, auf dem Sie verschiedene RSSFeeds aus dem Bereich Gaming lesen können. Abonnieren Sie am besten die wichtigen Gaming-Magazine und auf jeden Fall ein paar Gamer-Blogs. Darüber hinaus lohnen sich eine Hashtag-Suche auf Twitter und natürlich die Einrichtung von Google-Alerts $\mathrm{zu}$ verschiedenen Gaming-Themen. Schließlich lohnt sich Youtube hervorragend für das Thema Gaming. Hier finden Sie viele Let's Play-Videos und natürlich Interviews und Vorträge von Experten wie z. B. Katie Salen, Eli Neiburger oder Jane Mc-Gonigal. Sie können diese aus der passiven Nutzung gewonnenen Daten auch aktiv nutzen, indem Sie sie z. B. auf Facebook posten.

Probieren Sie unbedingt verschiedene Angebote und Plattformen aus. Finden Sie in Ruhe heraus, welche Inhalte und Angebote für Sie relevant sind. 